\title{
First test beam results of prototype modules for the upgrade of the ATLAS strip tracking detector
}

\author{
Susanne Kuehn, on behalf of the ATLAS Collaboration ${ }^{* *}$ \\ Albert-Ludwigs-University Freiburg, Germany, now at CERN, Geneva, Switzerland \\ E-mail: susanne.kuehnecern.ch
}

The LHC is foreseen to be upgraded to the High-Luminosity LHC (HL-LHC). This will result in higher particle rates and radiation doses. The ATLAS experiment plans to replace its inner tracking detector by a new all-silicon tracker which is based on the concept of modularity. For the new silicon strip tracker a large prototyping and evaluation campaign is ongoing. Many modules of different types were built and tested both in the laboratories and in test beams. In the following first results obtained in test beams are presented. Both mini and full-size modules for the central and forward regions were tested before and after irradiation to fluences as expected at the HLLHC.

38th International Conference on High Energy Physics

3-10 August 2016

Chicago, USA

\footnotetext{
* Speaker.

${ }^{\dagger}$ This work is supported by the European Social Fund and by the Ministry Of Science, Research and the Arts Baden-Wuerttemberg.
} 


\section{Introduction}

In several years the LHC is foreseen to be upgraded to the HL-LHC. This will result in instantaneous luminosities of up to $7.5 \times 10^{34} \mathrm{~cm}^{-2} \mathrm{~s}^{-1}$ and up to 200 simultaneous interactions per bunch crossing. In addition, high particle fluences require for example for the strip tracking detector to be able to withstand doses of $2 \times 10^{15} \mathrm{n}_{\mathrm{eq}} / \mathrm{cm}^{2}$ for an integrated luminosity of $3000 \mathrm{fb}^{-1}$ [1]. For high tracking performance, similar to the current tracking detector but under conditions of the HL-LHC, the ATLAS experiment [2] foresees to upgrade its tracking detector by an all-silicon detector [3]. An important task is prototyping and evaluating of detector components, especially before and after irradiation to fluences as expected at the HL-LHC. In the following paper electrical measurements and results from studies at particle test beams are presented. Conclusions are given on the performance of the modules.

\section{Layout and concept of the new inner tracker}

The upgraded all-silicon tracker of the ATLAS experiment, so-called Inner Tracker ITk will replace the current inner detector consisting of the silicon pixel, strip tracker and transition radiation tracker. It is laid out with five layers of pixel sensors and four layers of strip modules in the central region. In the forward region six layers of strip modules and a large amount of pixel rings covering values of $\eta$ of up to four are planned. Several layouts are under evaluation [4]. The silicon strip tracking detector will be built out of modules, consisting of readout boards with ASICs glued on top of silicon sensors. These modules will be assembled on carbon core structures.

\section{Prototyping of modules}

A large effort has been conducted in the prototyping of modules for both the barrel and forward region of the ITk with a series of prototypes being built. Modules consist of sensors with readout electronics glued on top. The sensors are n-in-p float-zone strip sensors with p-stop isolation and $74.5 \mu \mathrm{m}$ strip pitch. Their thickness is $320 \mu \mathrm{m}$ [5]. Their charge collection efficiency was measured using analog readout electronics both before and after irradiation with protons, gammas and neutrons. After beneficial annealing and irradiation to $2 \times 10^{15} \mathrm{n}_{\mathrm{eq}} / \mathrm{cm}^{2}$ of the order of 10 thousand electrons are collected [6] and [7]. The foreseen readout chips are binary and produced in the $130 \mathrm{~nm}$ CMOS process [8]. Depending on module type up to 20 ASICs are glued on a readout board (hybrid). Modules are powered in DC-DC mode for increased power efficiency. A module for the central region is shown on the left of Fig. 1. In addition to full-size modules, so-called mini modules were assembled. They use standard hybrids but a reduced number of ASICs and mini strip sensors of size $1 \mathrm{~cm}$ by $1 \mathrm{~cm}$. A photo of a mini module for the forward region is depicted on the right of Fig. 1.

\section{Methods for characterisation}

A range of modules were characterised in detail with the following methods. 

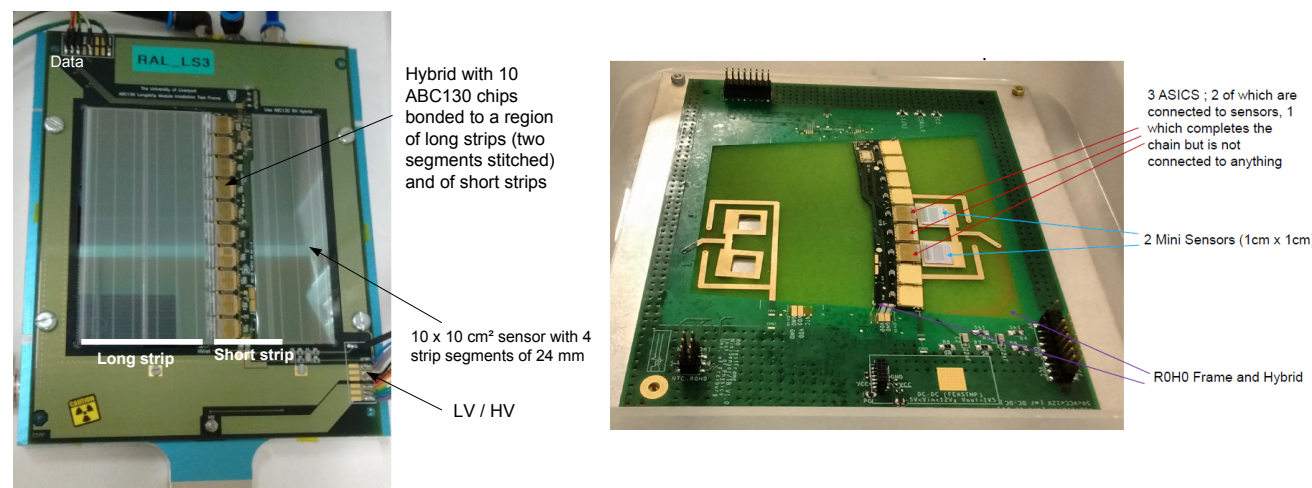

Figure 1: Photo of a silicon strip module for the central region (left) and of a mini module with a hybrid for the forward regions and two silicon strip mini sensors (right).

\subsection{Electrical tests}

Electrical performance of the modules was evaluated by injecting a range of input charges via the chip calibration circuitry, and measuring the module response via a series of threshold scans. Consequently the chip gain and input noise can be measured by performing a threshold scan. In addition, IV-curves were measured.

\subsection{Measurements at test beams}

Test beams are a powerful tool to determine detector parameters like efficiency, noise and collected charge using tracks of highly energetic particles. Several test beams were conducted in the collaboration. They were done at SLAC with $10 \mathrm{GeV}$ electrons, at DESY with $4.8 \mathrm{GeV}$ electrons and at CERN with $120 \mathrm{GeV}$ pions. For tracking, EUDET style telescopes with six pixel planes with about $3 \mu \mathrm{m}$ pointing resolution were used. An FE-I4 pixel detector was deployed as a timing plane (time resolution $25 \mathrm{~ns}$ ) [9]. Mounts in dark boxes which are placed on x-y-stages allowed cold tests (at about $-30^{\circ} \mathrm{C}$ ) and at different positions. Test beam measurements were conducted with an unirradiated central and forward mini modules at DESY. At a later stage eleven positions on an unirradiated full-size central module and irradiated mini modules were tested. The irradiated mini modules consist of either ASICs irradiated with x-rays to $1.4 \mathrm{Mrad}$ or sensors irradiated with neutrons to HL-LHC fluences. A test beam at CERN was used to measure three positions on an irradiated full-size central module. The characterisation of the devices occurred with externally triggered binary readout at a rate of 200 to $300 \mathrm{~Hz}$. The track reconstruction is performed by deploying a General Broken Lines algorithm and the EUTelescope software for reconstruction [10]. The raw data of the module and telescope was converted to a LCIO data format. Consequently, the EUTelescope code was used to extract clusters, telescope tracks and module hit positions. Custom analysis software was written to then calculate hit efficiencies of the devices under test.

\subsection{Irradiation of modules}

To understand the end of life performance of the detectors, it is important to measure the performance of modules after irradiation. A full-size module of the central region was mounted and 
powered (LV) and clocked during an irradiation with $24 \mathrm{GeV}$ protons in the PS at CERN. The irradiation was conducted cold (at about $-20^{\circ} \mathrm{C}$ ) and the total achieved fluence was $8 \times 10^{14} \mathrm{n}_{\mathrm{eq}} / \mathrm{cm}^{2}$. During irradiation a change of the current of the ASICs was observed. This behaviour as a function of dose is shown in Fig. 2. At low total ionising dose (TID) a sharp rise in current was observed, followed by a slow fall with increased TID. Several times the beam was off and led to decreases in current. The effect is under investigation within the collaboration to determine its dependence on dose rate and temperature.

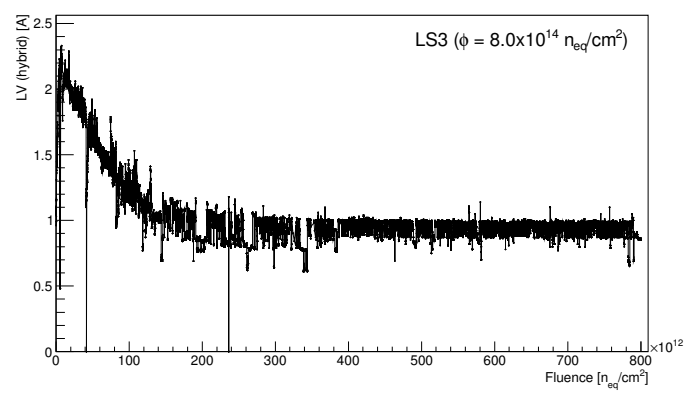

Figure 2: Low voltage current of the hybrid as a function of irradiation dose in $\mathrm{n}_{\mathrm{eq}} / \mathrm{cm}^{2}$ for the full-size module during the irradiation at the PS.

\section{Results}

\subsection{Results of electrical characterisation of modules}

The electrical characterisation resulted for the mini module of the forward region in noise values of unbonded channels of about 440 electrons and for channels bonded to sensor strips in about 540 electrons. Mini modules built from hybrids of the central region showed similar values of noise. In each module several channels were left unbonded to allow measurement of the ASIC noise level, and then comparison to the module noise for fully bonded channels. A full-size central module had noise values for strips of $2.4 \mathrm{~cm}$ length of about 750 electrons and for strips of double length of about 1090 electrons at a bias voltage of $400 \mathrm{~V}$ and with a leakage current of $0.2 \mu \mathrm{A}$ at room temperature. The noise levels were slightly higher than the expected performance predicted by chip simulation studies. This is believed to be caused by the non optimal tuning of the ASIC front-end amplifiers, and studies are ongoing to determine the correct tunings and subsequent lower noise levels.

After irradiation the module was fully functional and electrically tested. The I-V curve of the irradiated full-size module at different temperatures is given on the left of Fig. 3. Measurements of noise were also performed and more tests with optimised tuning are ongoing. First values are for the short strips about 920 electrons and for long strips about 1410 electrons.

\subsection{Results of test beam measurements}

An important result of the test beams is that all modules (both non- and post-irradiated) were successfully operated and tested in beams. The binary ASICs could be readout in the test beam infrastructure using external triggering. The correlation of hits of the module (local hits) and the tracks of the telescope (fitted hits in local system) is shown on the right of Fig. 3. 

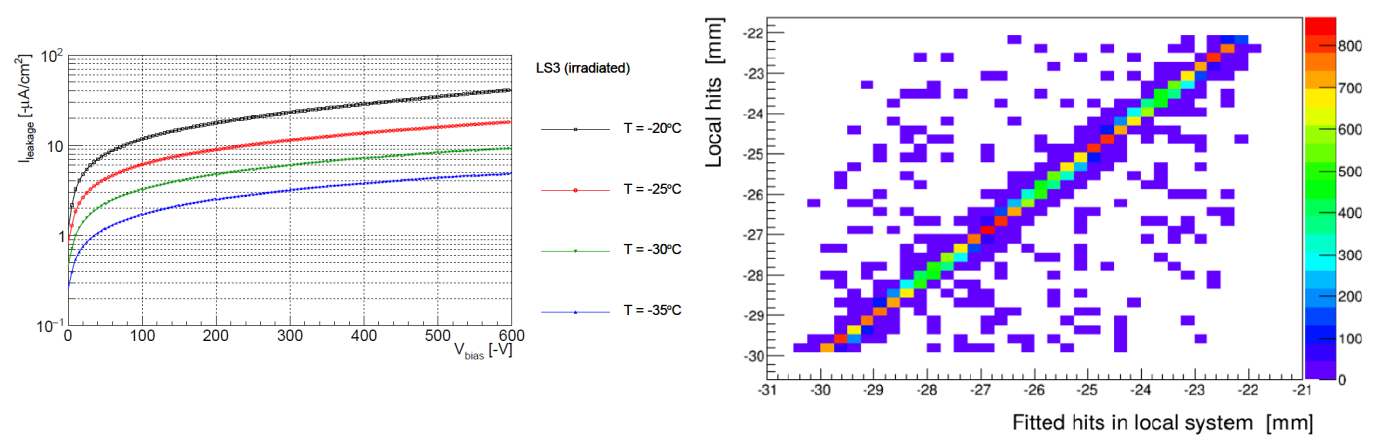

Figure 3: Leakage current of the irradiated full-size module as a function of bias voltage at different operation temperatures (left). Distribution of correlation of hits of the module (local hits) and the tracks of the telescope (fitted hits in local system) (right). The z-axis gives the number of hits.

\subsubsection{Mini module for the forward region}

For the mini modules of the forward region a residual of about $35 \mu \mathrm{m}$ was measured at a bias voltage of $350 \mathrm{~V}$, as displayed on the left of Fig. 4. This is slightly larger than expected from the pitch of $75 \mu \mathrm{m}$. It is believed to be caused by multiple scattering effects. In addition, the cluster size was measured at low thresholds, below the value of the most probable collected charge. It was found that the cluster size is around 1.5 between strips.
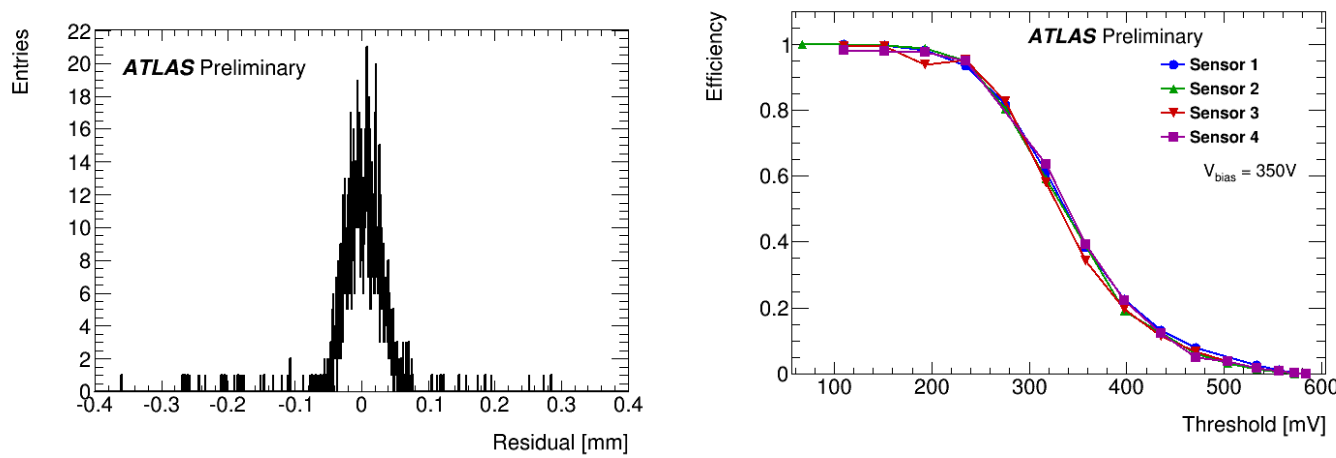

Figure 4: Distribution of residual for the mini module at a bias voltage of $450 \mathrm{~V}$ (left). Measured efficiency as a function of threshold in $\mathrm{mV}$ for four sensors of two mini modules (right).

\subsubsection{Mini module for the central region}

Two mini modules with four unirradiated sensors for the central region were investigated. The efficiency is determined as number of hits on the sensor over number of tracks in time in a region of $\pm 100 \mu \mathrm{m}$. For all mini modules similar distributions were measured, resulting in a similar amount of collected charge. The measured efficiency as a function of threshold at a bias voltage of $350 \mathrm{~V}$, above full depletion is given on the right of Fig.4. The calculation in absolute amount of charge is still ongoing.

\subsubsection{Full-size module for the central region}

For an unirradiated full-size module the residual was measured at different positions to values 
of 35-45 $\mu \mathrm{m}$. For both short and long strips a similar behaviour of collected charge was found. Preliminary estimates give a signal-to-noise ratio above 30 for both cases. Detailed analyses are ongoing to determine the absolute amount of signal.

\section{Summary}

For the upgrade of the tracking detector of the ATLAS experiment an all-silicon tracker is foreseen. It has a modular concept. The prototyping and measurements of detector components are heavily pursued. Several test beams were successfully conducted and tests of modules performed before and after irradiation to fluences as expected at the HL-LHC. Different mini modules for the central and forward region show a similar behaviour in noise measurements. In addition, measurements of unirradiated devices determine a high efficiency and high signal-to-noise ratio before irradiation. The evaluation of irradiated devices is still ongoing. A comparison to data collected with a beta source and from simulation has started.

\section{Acknowledgement}

This project has received funding from the European Union's Horizon 2020 Research and Innovation programme under Grant Agreement no. 654168. The measurements leading to these results have been performed at the Test Beam Facility at DESY Hamburg (Germany), a member of the Helmholtz Association (HGF).

\section{References}

[1] The ATLAS Collaboration, Fluence Plots for ITk Extended at4 layout, ITK-20016-002.

[2] The ATLAS Collaboration, G. Aad et al., The ATLAS Experiment at the CERN Large Hadron Collider, JINST 3 S08003 (2008).

[3] The ATLAS Collaboration, ATLAS Phase-II Upgrade Scoping Document, CERN-LHCC-2015-020, LHCC-G-166 (2015).

[4] The ATLAS Collaboration, Expected Performance of the ATLAS Inner Tracker at the High-Luminosity LHC, ATL-PHYS-PUB-2016-025.

[5] N. Unno et al., Development of n+-in-p large-area silicon microstrip sensors for very high radiation environments - ATLAS12 design and initial results, Nucl. Instrum. and Meth. A765 (2014) 80-90.

[6] K. Hara et al., Charge collection and field profile studies of heavily irradiated strip sensors for the ATLAS inner tracker upgrade, Nucl. Instrum. and Meth. A831 (2016) 181.

[7] R. Mori et al., Evaluation of the performance of irradiated silicon strip sensors for the forward detector of the ATLAS Inner Tracker Upgrade, Nucl. Instrum. and Meth. A831 (2016) 207.

[8] J. Kaplon, The ABCN Front-end Chip for ATLAS Inner Detector Upgrade, Topical Workshop on Electronics for Particle Physics (TWEPP 2008), pg. 116 [http://cdsweb.cern.ch/record/1158514].

[9] H. Jansen et al., Performance of the EUDET-type beam telescopes, EPJ Techn Instrum 3:7 (2016).

[10] T. Nelson et al., Track reconstruction and alignment for ATLAS Devices Under Test. DESY Note (2014). 